\title{
Similarity and the false recognition of prototypes*
}

\author{
ALAN S. LEVY and STANLEY HESHKA \\ Columbia University, New York, N.Y. 10027
}

Posner (1969) has reported studies using a continuous recognition task paradigm which he interprets as providing evidence that cognitive prototypes are extracted from sets of viewed patterns. An attempt to replicate and extend Posner's findings was only partly successful and drew attention to possible alternative explanations for the large number of "false alarms" elicited by certain patterns. The similarity of a pattern to immediately preceding exemplars appears to play a crucial role in eliciting false alarms. Until studies are available which explicitly control for similarity, the interpretation of continuous recognition task results must remain uncertain.

In a recent publication, Posner (1969) reports a study which he interprets as providing supporting evidence for the notion that the process of category recognition involves a prototype which is extracted from the experienced set of exemplars. The study purports to demonstrate that the prototype acquires a cognitive status similar to that of the exemplars which the $S$ has actually seen. To demonstrate the status of such a prototype, Posner's study employed the continuous recognition task paradigm (Shepard \& Teghtsoonian, 1961). Ss are required to say whether or not a particular exemplar has appeared before in a series by responding "old" or "new." After a number of exemplars have been shown, the prototype, from which the exemplars have been generated by a process of random distortion, is presented. Posner reports that the probability of identifying the prototype as already having been seen when, in fact, it has not appeared before in the series, i.e., the probability of responding "old" to the prototype $[\mathrm{P}(\mathrm{O}$ |prototype $)]$, is the same as the probability of correctly recognizing an exemplar which has appeared before in the series, i.e., the probability of responding "old" to an old pattern $[\mathrm{P}(\mathrm{O} \mid \mathrm{O})]$. This is cited as evidence that the prototype, which has not been seen, has been abstracted from the distortions and has acquired a cognitive status similar to that of experienced exemplars.

The recognition task may involve two distinct processes: correctly identifying a test pattern as "old" when it has been presented before and incorrectly responding "old" to a pattern that has not been presented in the series (a "false alarm"). The former process, successful recognition, presumably depends on a match between the viewed pattern and some stored representation of it. However, in the case of false alarms,

*We would like to thank Robert M. Krauss for his comments and suggestions on data analysis and Stephen Woods for sponsoring and taking editorial responsibility for the paper. this cannot be the process since the $S$, not having seen the exemplar, has no cognitive representation of the pattern. One obvious possibility which suggests itself is that the likelihood of false alarms to any new pattern, including the prototype, will be a direct function of the similarity between the test pattern and recently viewed exemplars. Posner's argument states, however, that the prototype produces false alarms because it has a corresponding cognitive representation and not simply because of fortuitous similarity with representations of preceding patterns. Now, it is true and unavoidable that if we consider the whole series of exemplars, the prototype, because of the method of construction, is the "average" pattern and thus, in this sense, the most similar pattern possible. We are concerned, however, with the similarity relation between the target and the three or four immediately preceding patterns, since these will have the most salient representation. In this sense, the prototype as a statistical average is not necessarily more similar to the preceding patterns than just some other new pattern. It becomes important, if we are to interpret the results unambiguously, that the prototype not have a greater local similarity than other control targets that are also new. Posner has recognized this problem and has tried to deal with it by including, along with the prototype target, other new patterns as controls which he claims are as similar to the experienced exemplars as is the prototype. Unfortunately, he does not actually measure similarity but simply claims that the control patterns are drawn from a population which, on the average, is just as different from already seen exemplars as is the prototype. We were not entirely satisfied with this control technique and added to the procedure a measure of similarity so that we could look at the contribution of similarity to the phenomenon of the "familiar" prototype.

It also seemed likely that the probability of responding "old" to the prototype might vary with certain parameters of the series of distortions, e.g., amount of variability among patterns in the series, number of exemplars presented, and exposure time for the patterns. In order to test whether Posner's finding would replicate when these parameters were varied, an experiment was run using the continuous recognition paradigm

\section{METHOD}

Two series of distortions were presented to 27 Ss. Ss were drawn from the introductory psychology $S$ pool and were partially fulfilling their experimental requirement. The prototype was different for each series and was a random computer-generated nine-dot pattern (Posner, Goldsmith, \& Welton, 1967). ${ }^{1}$ In the first series, the low variability set, 17 5-bit/dot distortions were presented; in the second series, the high variability set, 12 - and 7.7-bit/dot distortions were used. 
Table 1

Conditional Probabilities for the Recognition of a Pattern as Old

\begin{tabular}{ccccc} 
Series & $\overline{\mathrm{P}}(\mathrm{O} \mid \mathrm{O})$ & $\overline{\mathrm{P}}(\mathrm{O} \mid \mathrm{N})$ & $\mathrm{P}(\mathrm{O} \mid$ Proto) & $\begin{array}{c}\text { Likelihood } \\
\text { Ratio }\end{array}$ \\
\hline 1 & .70 & .47 & .41 & 1.47 \\
2 & .60 & .14 & .48 & 4.29 \\
\hline
\end{tabular}

After the first two exemplars, old and new patterns were randomly interspersed in both series in a ratio of approximately .5. Patterns were presented to Ss using an automatic slide projector, with an exposure time of $8 \mathrm{sec}$.

A brief series of distortions taken from a third prototype was presented at the beginning of the session to ensure that Ss understood the instructions and to familiarize them with the task. Ss were told to identify each pattern shown as either old or new and to indicate their decision on the sheet provided by writing $\mathrm{O}$ or $\mathrm{N}$.

\section{RESULTS}

The dependent measure calculated over all Ss for each dot pattern was the probability that a dot pattern was seen as old by our $\mathrm{Ss}, \mathrm{P}(\mathrm{O})$. In addition, we calculated two conditional probabilities, $\mathrm{P}(\mathrm{O} \mid \mathrm{O})$ and $\mathrm{P}(\mathrm{O} \mid \mathrm{N})$, for each series. These measures, along with the probability of responding "old" to the prototype, $\mathrm{P}(\mathrm{O}$ |prototype), constitute the major results of the study. The results are presented in Table 1.

These results indicate that the probability of responding "old" to the prototype is not the same as the probability of responding "old" to patterns that have been seen before. In fact, it does not even seem that the prototype is necessarily seen as more familiar than other new patterns. In Series 1 there were several new patterns for which $\mathrm{P}(\mathrm{O} \mid \mathrm{N})$ was over .80 , and one new pattern was called "old" by all 27 Ss.

One important difference between Series 1 and Series 2 which may have affected the Ss' responses to the prototype is the difficulty of making the required discriminations between old and new patterns. The likelihood ratios, $\mathrm{P}(\mathrm{O} \mid \mathrm{O}) / \mathrm{P}(\mathrm{O} \mid \mathrm{N})$, which are an index of how well Ss perform the task, are quite different for the two series (see Table 1). It seems that when variability among exemplars is high, as in Series 2 , and the discriminations are easier, the prototype is seen as more familiar than other new patterns.

Still another explanation is suggested by examining the relation of similarity to false alarms. An index of similarity between dot patterns in a series was calculated by taking the ordered set of interdot distances for a dot pattern and computing the correlation coefficient for each pair of patterns. This correlation is a good quantitative index of the similarity between two dot patterns, and the best measure of similarity between a target and the previously seen exemplars will be some function of the pairwise similarities. On the assumption that the effect of similarity would decrease with temporal distance and number of intervening exposures, we first calculated the average similarity, $S$, for each pattern with the three immediately preceding exemplars.
Table 2 presents the averages of these similarities, $\bar{S}$, for old and new patterns in each series, as well as the similarity for each prototype. An examination of Table 2 suggests that only when the prototype is more similar to its immediately preceding exemplars than new patterns throughout the series are to their immediately preceding exemplars is it seen as more "familiar."

This suggests an alternative explanation for the familiarity of the prototype which does not require postulating an abstracted cognitive representation. If this proposal is valid, then we could expect a strong relation between similarity and false alarms throughout the series. We calculated the correlation between our index of similarity, S, and the probability of responding "old" to a new pattern, $\mathrm{P}(\mathrm{O} \mid \mathrm{N})$, over all "new" patterns in the series, prototype included. For Series $1 \mathrm{r}=.62$ and for Series $2 \mathrm{r}=.79$. It would appear that, for both series, even using an ad hoc index, similarity is significantly related to false alarms. On the other hand, the correlation between similarity and recognition of old patterns, $\mathrm{P}(\mathrm{O} \mid \mathrm{O})$, is .02 for Series 1 and .09 for Series 2 . Thus, similarity seems to be involved only with false recognition of new patterns.

We also used our pairwise similarities as predictors in a stepwise multiple regression, with $P(O \mid N)$ as the dependent variable. Conceptually this replaces our ad hoc "average" similarity of a pattern with the preceding three exemplars, with the maximizing regression coefficients generated by the multiple regression. Excellent predictions were obtained for $\mathrm{P}(\mathrm{O} \mid \mathrm{N})$ using similarity measures with each of the five preceding exemplars as predictors. The total regression equation for Series 1 accounted for $71 \%$ of the variance and the comparable figure for Series 2 was $93 \%$. (These values should be interpreted cautiously because of the small number of cases involved.) Not all similarities had equal predictive value. Similarity with the pattern immediately preceding the target seemed to be discounted, probably because a very accurate comparison is possible between the target and the pattern just seen. The next pattern was most important as a predictor, then predictive value declined as distance from test pattern increased.

All this strongly suggests that local similarity is an important factor in false alarms and that, to the extent that a prototype has a high local similarity with preceding patterns, we should be cautious in interpreting the high probability of false alarms to the prototype.

Table 2

Measure of Similarity Between the Target and the Three Preceding Exemplars

\begin{tabular}{cccc}
\hline Series & $\overline{\mathrm{S}}(\mathrm{O})$ & $\overline{\mathrm{S}}(\mathrm{N})$ & $\mathrm{S}$ (Proto) \\
\hline 1 & .9393 & .9164 & .9170 \\
2 & .7888 & .7996 & .8853 \\
\hline
\end{tabular}




\section{DISCUSSION}

It would seem that the continuous recognition procedure does not always yield the "familiar prototype" phenomenon and that even when it does there is some question as to the basis for this familiarity. Posner's original claim that a representation of the prototype is abstracted from the set of exemplars assumes that randomly selected new patterns are adequate control comparisons for the prototype. We find that there are instances when new patterns other than the prototype are also seen as highly familiar, and this seems to be explained most easily by the high similarity these patterns have with the preceding few exemplars. This suggests that false recognition is mainly a function of local similarity. Since prototypes will tend to have greater local similarity, there is no need to postulate a special mechanism for their recognition unless it can be shown that local similarity does not account for the results. Given the rules and probabilities for construction distortions set out in Posner et al (1967, pp. 29-31), the probability that a preceding 7.7-bit distortion will be more similar to the prototype than to another randomly selected 7.7-bit distortion used as a control is greater than .70. This makes the control procedure used by Posner clearly inadequate. The appropriate controls must be new distortions which are carefully selected so that they are no less similar to preceding patterns than is the prototype.

Furthermore, our results suggest that seeing a prototype as old is quite different from recognizing a truly old pattern as old. The response "old" to a pattern that has not been viewed before seems to be related to its local similarity; recognition of old patterns is independent of this parameter. This suggests that the recognition process may be different for old and new patterns. At the least, our findings indicate that future studies along these lines should not overlook the contribution of similarity to false recognition.

\section{REFERENCES}

Posner, M. I. Abstraction and the process of recognition. Psychology of Learning \& Motivation, 1969, 3, 43-100.

Posner, M. I., Goldsmith, R., \& Welton, K. E. Perceived distance and the classification of distorted patterns. Journal of Experimental Psychology, 1967, 73, 28-38

Shepard, R. N. \& Teghtsoonian, M. Retention of information under conditions approaching a steady state. Journal of Experimental Psychology, 1961, 62, 302-309.

\section{NOTE}

1. We want to especially thank Donald Elman who wrote the computer program for the production of dot patterns.

(Received for publication November 29, 1972.) 